\title{
openheart Is 6-month GRACE risk score a useful tool to predict stroke after an acute coronary syndrome?
}

\author{
Belén Álvarez-Álvarez, Sergio Raposeiras-Roubín, Emad Abu-Assi, \\ Cristina Cambeiro-González, Santiago Gestal-Romaní, Andrea López-López, \\ Noelia Bouzas-Cruz, María Castiñeira-Busto, Ozoda Saidhodjayeva, \\ Alfredo Redondo-Diéguez, Eva Pereira López, José María García-Acuña, \\ José Ramón González-Juanatey
}

To cite: Álvarez-Álvarez B, Raposeiras-Roubín S,

Abu-Assi E, et al. Is 6-month GRACE risk score a useful tool to predict stroke after an acute coronary syndrome?. Open Heart 2014;1:e000123. doi:10.1136/openhrt-2014000123

Received 13 March 2014 Revised 13 August 2014 Accepted 4 November 2014

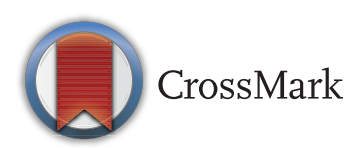

Department of Cardiology, University Clinical Hospital of Santiago de Compostela, Santiago de Compostela, Spain

Correspondence to Belén Álvarez Álvarez; belenalvarez85@hotmail.com

\section{ABSTRACT}

Objectives: The risk of stroke after an acute coronary syndrome (ACS) has increased. The aim of this study was to do a comparative validation of the 6 -month GRACE (Global Registry of Acute Coronary Events) risk score and $\mathrm{CH}_{2} \mathrm{DS}_{2}$ VASc risk score to predict the risk of post-ACS ischaemic stroke.

Methods: This was a retrospective study carried out in a single centre with 4229 patients with ACS discharged between 2004 and $2010(66.9 \pm 12.8$ years, $27.9 \%$ women, $64.2 \%$ underwent percutaneous coronary intervention). The primary end point is the occurrence of an ischaemic stroke during follow-up (median 4.6 years, IQR 2.7-7.1 years).

Results: $184(4.4 \%)$ patients developed an ischaemic stroke; $153(83.2 \%)$ had sinus rhythm and 31 (16.9\%) had atrial fibrillation. Patients with stroke were older, with higher rates of hypertension, diabetes, previous stroke and previous coronary artery disease. The HR for $\mathrm{CHA}_{2} \mathrm{DS}_{2} \mathrm{VASc}$ was $1.36(95 \% \mathrm{Cl}, 1.27$ to $1.48, \mathrm{p}<0.001)$ and for GRACE, HR was $1.02(95 \% \mathrm{Cl}, 1.01$ to 1.03 , $\mathrm{p}<0.001)$. Both risk scores show adequate discriminative ability (c-index $0.63 \pm 0.02$ and $0.60 \pm 0.02$ for $\mathrm{CHA}_{2} \mathrm{DS}_{2}$ VASc and GRACE, respectively). In the reclassification method there was no difference (Net Reclassification Improvement 1.98\%, $\mathrm{p}=0.69$ ). Comparing moderate-risk/high-risk patients with low-risk patients, both risk scores showed very high negative predictive value $\left(98.5 \%\right.$ for $\mathrm{CHA}_{2} \mathrm{DS}_{2} \mathrm{VASc}$, $98.1 \%$ for GRACE). The sensitivity of $\mathrm{CHA}_{2} \mathrm{DS}_{2} \mathrm{VASc}$ score was higher than the GRACE risk score (95.1\% vs $87.0 \%)$, whereas specificity was lower ( $14.4 \%$ vs $30.2 \%$ ).

Conclusions: The 6-month GRACE model is a clinical risk score that facilitates the identification of individual patients who are at high risk of ischaemic stroke after ACS discharge.

\section{INTRODUCTION}

Stroke after an acute coronary syndrome (ACS) is a rare complication. Although its incidence is higher in the first few days after ACS, ${ }^{1}$ the risk of stroke continues with the

\section{KEY QUESTIONS}

What is already known about this subject?

- Patients, who survive an acute coronary syndrome (ACS), have an increased risk of stroke and therefore, a greater possibility of mortality. Most studies have assessed the incidence and predictors during admission, and during the first year of stroke post-ACS. Although there has been recently a reduction in incidence of strokes due to the advances in treatment and secondary prevention; 6-month GRACE (Global Registry of Acute Coronary Events) risk score is a useful tool to predict mortality and reinfarction. It is, however, not well defined in its ability to predict stroke after an ACS. Owing to the consequences of having an ACS, we propose this trial to assess the 6-month GRACE risk score in this setting.

What does this study add?

- Six-month GRACE risk score estimates mortality after discharge. This proved useful to determine the long-term risk of thrombotic events and was validated in large external data sets. So far, no studies have predicted the risk of ischaemic stroke after ACS. In our study, we want to conduct a comparative validation of the 6-month GRACE risk score to predict the risk of post-ACS ischaemic stroke.

How might this impact on clinical practice?

- Clinical practice does not have a useful tool to predict the risk of ischaemic stroke after ACS. It is important for the existence of a validated riskstandardised model to identify high-risk patients. Our study adds another utility to the 6-month GRACE risk score when it predicts thromboembolic risk. Then, 6-month GRACE risk score allows us to predict mortality, reinfarction and ischaemic stroke risk in the follow-up.

follow-up. Despite antithrombotic therapy, recent studies have shown a long-term rate of stroke after an ACS, between $1 \%$ and $4 \% .^{2-7}$ 
Also the consequences of this complication are very serious. Owing to this, it is important to identify the patients with increased risk of stroke.

To date, there are two scores that allow us to estimate the thromboembolic risk of stroke in non-valvular atrial fibrillation (AF): $\mathrm{CHADS}_{2}{ }^{8}$ and later, $\mathrm{CHA}_{2} \mathrm{DS}_{2}$ VASc. $^{9}$ Although these two risk scores were not specifically designed to predict stroke risk after an ACS, they were recently validated in this setting. ${ }^{10}$ In addition to these, there are several scores to estimate the thrombotic risk after an ACS. The GRACE (Global Registry of Acute Coronary Events) risk score is the most extended, and it has been validated to predict death and reinfarction after an ACS. ${ }^{11-13}$

The aim of our study was to compare both risk scores, $\mathrm{CHA}_{2} \mathrm{DS}_{2} \mathrm{VASc}$ and 6-month GRACE risk score, to predict the long-term incidence of ischaemic stroke after an ACS.

\section{METHODS}

\section{Data sources and samples}

This was a retrospective study in which demographic, clinical and angiographic data, as well as data on management and in-hospital complications, had been prospectively collected and recorded in an electronic database. All patients with a diagnosis of ACS, admitted consecutively into our hospital between January 2004 and June 2010 were included in the study. ACS diagnosis was validated if the patient had new onset symptoms consistent with cardiac ischaemia and at least one of the following criteria: cardiac biomarkers above the higher normal laboratory limit, ST-segment deviation on ECG, inhospital stress testing showing ischaemia or a known history of coronary vessel disease. Patients were classified as having acute myocardial infarction (AMI) with ST-segment elevation myocardial infarction (STEMI) or non-ST-segment elevation ACS (NSTE-ACS; unstable angina and non-ST-segment elevation AMI). The initial cohort consisted of 4645 patients; 274 patients died during the inhospital phase. Of the 4371 discharged patients, we excluded those patients in whom ACS was precipitated in the context of surgery, sepsis, trauma or cocaine consumption $(n=41)$, and those with missing data for any variable of GRACE risk score $(n=67)$. Of the 4263 remaining patients, 1-month follow-up was completed for $99.2 \%$ (34 patients without follow-up data). Thus, the final cohort was composed of 4229 patients. The study complies with the Declaration of Helsinki and was approved by the Clinical Research Ethics Committee of our hospital.

\section{End point}

The primary end point of this study was the occurrence of an ischaemic stroke during follow-up (median 4.6 years, IQR 2.7-7.1 years), confirmed through cerebral CT when deemed necessary by the attending neurologist. Patients were followed for a mean of 4.6 years (IQR 2.7-
7.1 years) after their discharge. Methods of follow-up involved one or more of the following: use of hospital records, hospital visits, phone call to the patient's general physician and/or phone call to the patient.

\section{GRACE risk score calculation}

The 6-month GRACE risk score was calculated for each patient by assigning the appropriate number of points for each of the nine prognostic variables that enter into the calculation: age, history of heart failure, history of AMI, heart rate and systolic blood pressure at admission, ST-segment depression, serum creatinine at admission, elevated myocardial necrosis markers or enzymes and lack of percutaneous coronary revascularisation during admission (Supplemental Data). Three risk categories were established using the cut-off points set out in the GRACE study. Therefore, in the low-risk category, the GRACE score was 27-99 points for STEMI and 1-88 for NSTE-ACS; in the intermediate risk category, the score for STEMI was 100-127 and 89-118 for NSTE-ACS; and in the high risk category, the score for STEMI was 128-263 and 119-263 for NSTE-ACS.

\section{Statistical analysis}

All analyses were performed using SPSS (V.17.0, SPSS Inc, Chicago, Illinois) and STATA V.13.0. Discrete variables are expressed as frequencies and percentages, and quantitative data are presented as the mean \pm SD. $\chi^{2}$ Test was used to compare discrete variables and the Student $t$ test to compare quantitative variables. The correlation between the risk scores was performed using Pearson test. A Cox proportional hazards model was used to estimate the $\mathrm{HR}$ and $95 \% \mathrm{CI}$ of each variable in the GRACE and $\mathrm{CHA}_{2} \mathrm{DS}_{2}$ VASc risk scores. Both stroke and death before ACS occurrence were regarded as competing risks using sub-HR (SHR). ${ }^{14}$ Cumulative stroke rates were analysed by the method of Kaplan-Meier (Log-rank test) for the different risk groups.

The c-index $^{15}$ has been used as the measure for model discrimination, equivalent to the area under a receiver operating characteristic curve; this was used to determine the performance of GRACE and $\mathrm{CHA}_{2} \mathrm{DS}_{2} \mathrm{VASc}$ risk scores in predicting the follow-up stroke. Negative and positive predictive values for GRACE risk score were also computed for the moderate-risk/high-risk group versus low-risk group.

We also analyse the risk reclassification with Pencina's method. ${ }^{16}$ This allowed us to calculate the Net Reclassification Improvement (NRI). It is a fraction of net reclassification based on predictions with or without a marker, and it improves the sensitivity and specificity. A $p$ value $<0.05$ was considered statistically significant.

\section{RESULTS}

\section{Baseline characteristics and events}

A total of 4229 patients were included in the analysis. The baseline characteristics are shown in table 1 . The median 
Table 1 Clinical characteristics, in-hospital management and treatment at discharge

\begin{tabular}{|c|c|c|c|c|}
\hline Variables & Total population & Stroke & No stroke & p Value \\
\hline Age (years) & $66.9 \pm 12.8$ & $71.3 \pm 10.2$ & $66.7 \pm 12.9$ & $<0.010$ \\
\hline Female sex & $27.9 \%$ & $33.2 \%$ & $27.6 \%$ & 0.101 \\
\hline GRACE 6 months & $112.9 \pm 33.4$ & $123.8 \pm 30.5$ & $112.5 \pm 33.5$ & $<0.010$ \\
\hline $\mathrm{CHA}_{2} \mathrm{DS}_{2} \mathrm{VASc}$ & $2.7 \pm 1.9$ & $3.4 \pm 1.8$ & $2.6 \pm 1.8$ & $<0.010$ \\
\hline MDRD-4 (mL/min/1.73 $\left.\mathrm{m}^{2}\right)$ & $74.9 \pm 39.3$ & $68.8 \pm 23.7$ & $75.1 \pm 39.8$ & 0.032 \\
\hline TNI peak (ng/mL) & $28.9 \pm 123.8$ & $20.8 \pm 40.6$ & $29.3 \pm 126.2$ & 0.366 \\
\hline Hypertension & $57.1 \%$ & $66.3 \%$ & $56.7 \%$ & 0.010 \\
\hline Diabetes & $26.5 \%$ & $35.9 \%$ & $26.0 \%$ & 0.003 \\
\hline Hypercholesterolemia & $45.2 \%$ & $46.2 \%$ & $45.1 \%$ & 0.779 \\
\hline Previous CAD & $23.1 \%$ & $29.9 \%$ & $22.8 \%$ & 0.026 \\
\hline Previous stroke & $6.8 \%$ & $14.1 \%$ & $6.5 \%$ & $<0.001$ \\
\hline \multicolumn{5}{|l|}{ Hospitalisation } \\
\hline$E F<40 \%$ & $12.8 \%$ & $14.1 \%$ & $12.7 \%$ & 0.576 \\
\hline STEMI & $31.5 \%$ & $27.7 \%$ & $31.7 \%$ & 0.256 \\
\hline Multivessel & $37.4 \%$ & $32.1 \%$ & $37.7 \%$ & 0.124 \\
\hline $\mathrm{PCl}$ & $64.2 \%$ & $56.5 \%$ & $64.5 \%$ & 0.027 \\
\hline \multicolumn{5}{|l|}{ At discharge } \\
\hline Dual antiplatelet therapy & $71.1 \%$ & $65.2 \%$ & $71.3 \%$ & 0.074 \\
\hline Warfarin & $7.4 \%$ & $10.9 \%$ & $7.2 \%$ & 0.062 \\
\hline ACEI/A2 blocker & $60.3 \%$ & $60.3 \%$ & $60.3 \%$ & 0.999 \\
\hline Statins & $83.3 \%$ & $79.3 \%$ & $83.5 \%$ & 0.141 \\
\hline$\beta$-Blocker & $67.7 \%$ & $66.3 \%$ & $67.8 \%$ & 0.669 \\
\hline
\end{tabular}

Values are $n(\%)$ or median (IQR).

MDRD-4, glomerular filtration rate calculated by MDRD Formula for glomerular filtration rate.

A2 blocker, angiotensin II receptor blocker; ACEI, ACE inhibitor; CAD, coronary artery disease; EF, ejection fraction; GRACE, Global Registry of Acute Coronary Events; MDRD, modification of diet in renal disease; PCl, percutaneous coronary intervention; STEMI, ST-segment elevation myocardial infarction; TNI, troponin.

of follow-up was 4.6 years, IQR 2.7-7.1 years. The median time for the stroke was $3.3 \pm 2.4$ years since ACS. During this period $184(4.4 \%)$ patients developed an ischaemic stroke, $153(83.15 \%)$ had sinus rhythm and $31(16.85 \%)$ had AF. Patients with stroke were older, with higher rates of hypertension, diabetes, a previous stroke and previous coronary artery disease.

\section{$\mathrm{CHA}_{2} \mathrm{DS}_{2}$ VASC and GRACE risk scores}

The $\mathrm{CHA}_{2} \mathrm{DS}_{2} \mathrm{VASc}$ and GRACE risks scores have demonstrated a strong correlation $(\mathrm{r}=0.685, \mathrm{p}<0.001)$. The discrimination of both risks scores to predict primary end point was adequate (c-index, $0.63 \pm 0.02$ and $0.60 \pm 0.02$ for $\mathrm{CHA}_{2} \mathrm{DS}_{2} \mathrm{VASc}$ and GRACE risks scores, respectively).

The HR to predict follow-up post-ACS ischaemic stroke was 1.36 (95\% CI 1.27 to $1.48, \mathrm{p}<0.001)$ for $\mathrm{CHA}_{2} \mathrm{DS}_{2} \mathrm{VASc}$ score and 1.02 (95\% CI, 1.01 to 1.03 , $\mathrm{p}<0.001)$ for GRACE risk score. In table 2 , the HR of each variable included in the $\mathrm{CHA}_{2} \mathrm{DS}_{2} \mathrm{VASc}$ and GRACE score was analysed.

Both risk scores demonstrated a risk gradient to predict post-ACS stroke: $1.5 \%, 2.7 \%$ and $5.3 \%$ for low, moderate and high $\mathrm{CHA}_{2} \mathrm{DS}_{2} \mathrm{VASc}$ risk groups, respectively, and $1.9 \%, 4.8 \%$ and $5.8 \%$ for low, moderate and high GRACE risk groups, respectively. Figure 1 illustrates Kaplan-Meier curves for ischaemic strokes during the follow-up according to the risk stratification.
The negative predictive values of $\mathrm{CHA}_{2} \mathrm{DS}_{2} \mathrm{VASc}$ and GRACE risks scores were $98.48 \% \quad(97-99.3 \%)$ and 98.07\% (97.1-98.7\%), respectively. After a reclassification analysis, there was no significant improvement in the global reclassification (NRI 1.98\%, $\mathrm{p}=0.69$ ).

The analysis, controlled for competitive events (death and stroke), confirmed that $\mathrm{CHA}_{2} \mathrm{DS}_{2} \mathrm{VASc}$ and GRACE risk scores were powerful predictors of stroke incidence during the follow-up (SHR 1.25; 95\% CI 1.16 to 1.34; $\mathrm{p}<0.001$, and HR 1.01; $95 \%$ CI 1.01 to $1.02 ; \mathrm{p}<0.001$, respectively).

\section{DISCUSSION}

We performed a comparative validation of GRACE and $\mathrm{CHA}_{2} \mathrm{DS}_{2} \mathrm{VASc}$ risk scores to predict ischaemic stroke after ACS. The main clinical finding of our study was that the discriminative ability of GRACE risk score to predict the primary end point was similar to $\mathrm{CHA}_{2} \mathrm{DS}_{2} \mathrm{VASc}$, even in patients with $\mathrm{AF}$.

Although the GRACE risk score was validated to quantify the risk of mortality and reinfarction in the acute phase $^{17}$ or in the follow-up, ${ }^{11}{ }^{12}$ little is known about its usefulness in predicting post-ACS stroke. ${ }^{18}$ Our study provides new evidence in this setting.

Stroke, a relatively rare complication after ACS, is associated with high mortality. ${ }^{5} 18$ It may occur due to a multitude of reasons, such as atherosclerotic disease and 
Table 2 HR of each variable of GRACE and $\mathrm{CHA}_{2} \mathrm{DS}_{2} \mathrm{VASc}$

\begin{tabular}{|c|c|c|c|}
\hline Variables & HR & $95 \% \mathrm{Cl}$ & $\begin{array}{l}p \\
\text { Value }\end{array}$ \\
\hline \multicolumn{4}{|l|}{ GRACE } \\
\hline Age & 1.048 & 1.034 to 1.062 & $<0.001$ \\
\hline $\begin{array}{l}\text { History of congestive } \\
\text { heart failure }\end{array}$ & 1.748 & 1.234 to 2.478 & 0.002 \\
\hline $\begin{array}{l}\text { History of myocardial } \\
\text { infarction }\end{array}$ & 1.359 & 0.909 to 2.032 & 0.135 \\
\hline Heart rate & 1.006 & 0.999 to 1.012 & 0.070 \\
\hline Systolic blood pressure & 1.005 & 1.001 to 1.010 & $<0.001$ \\
\hline ST-segment depression & 0.955 & 0.715 to 1.275 & 0.755 \\
\hline Creatinine & 1.279 & 1.105 to 1.481 & 0.001 \\
\hline $\begin{array}{l}\text { Elevated cardiac } \\
\text { markers }\end{array}$ & 1.197 & 0.813 to 1.762 & 0.362 \\
\hline No in-hospital PCI & 1.563 & 1.168 to 2.093 & 0.003 \\
\hline \multicolumn{4}{|l|}{$\mathrm{CHA}_{2} \mathrm{DS}_{2} \mathrm{VASc}$} \\
\hline C & 1.858 & 1.315 to 2.626 & $<0.001$ \\
\hline $\mathrm{H}$ & 1.629 & 1.200 to 2.211 & 0.002 \\
\hline$A_{2}$ & 2.723 & 1.880 to 3.945 & $<0.001$ \\
\hline$A_{1}$ & 2.245 & 1.544 to 3.264 & $<0.001$ \\
\hline $\mathrm{D}$ & 1.849 & 1.375 to 2.487 & $<0.001$ \\
\hline $\mathrm{S}_{2}$ & 2.844 & 1.877 to 4.309 & $<0.001$ \\
\hline Vasc & 1.734 & 1.291 to 2.330 & $<0.001$ \\
\hline Female sex & 1.327 & 0.976 to 1.804 & 0.071 \\
\hline
\end{tabular}

$A_{1}$, age between 65 and 74 years; $A_{2}$, age $\geq 75$ years; $C$, congestive heart failure; $D$, diabetes; $H$, hypertension; GRACE, Global Registry of Acute Coronary Events; $\mathrm{PCl}$, percutaneous coronary intervention; $\mathrm{S}_{2}$, stroke; Vasc, vascular disease.
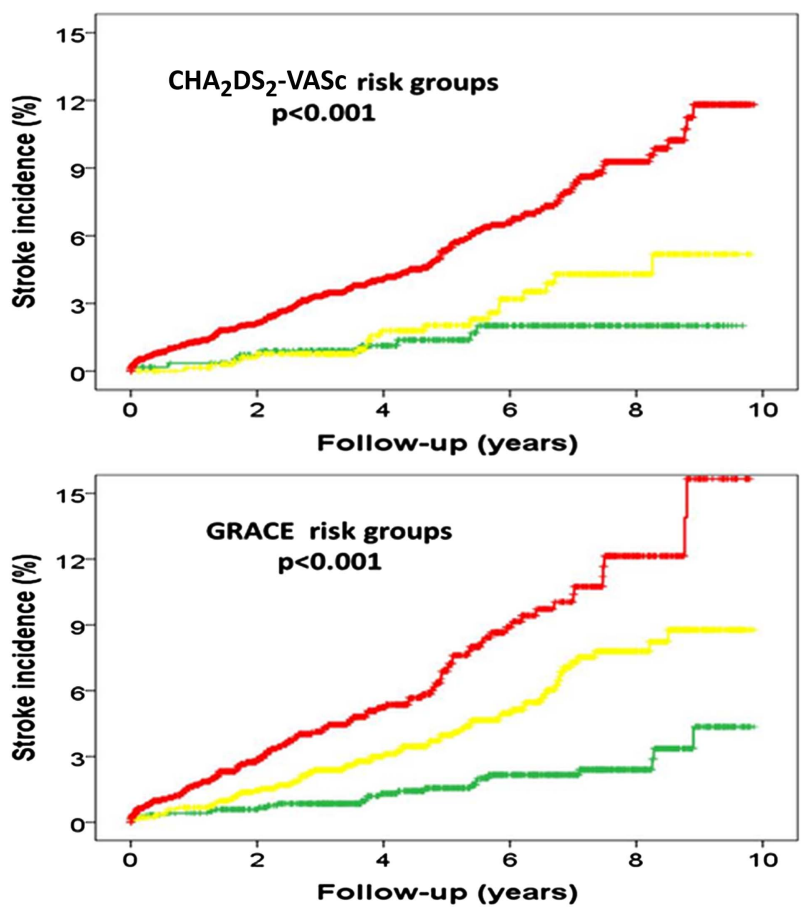

Figure 1 Kaplan-Meier curves illustrating survival according to CHA2DS2-VASc and GRACE risk scores. Green: low risk, yellow: moderate risk and red: high risk. thromboembolic events. Stroke represents one of the major causes of morbidity among hospital survivors of ACS. The identification of predictors for stroke in patients with ACS may help to optimise the treatment of high-risk patients. This could prevent fatal postdischarge consequences.

The incidence of stroke post-ACS has been estimated in several trials. ${ }^{19}$ This is highest in the first 5 days, ${ }^{1}$ and subsequently it is reduced. ${ }^{18}{ }^{20}$ Most of studies had assessed the incidence of stroke after ACS during the in-hospital phase, at 30 days or in the first year. In recent years, there have been some studies about the trends in the incidence of post-ACS stroke. Brammas et al published a reduction in the incidence of mortality in patients with post-ACS stroke, in the past few years, due to the improved treatment based on evidence. ${ }^{19}$

Clinical practice guidelines recommended risk stratification in ACS, ${ }^{21} 22$ in the acute phase and after hospital discharge, to improve the prognosis of these patients. To date, we have several risk scores to estimate the risk of thrombotic and thromboembolic events in ACS and AF, respectively. These risk scores have been developed and validated in the past years.

The 6-month GRACE ${ }^{11}$ risk score is a simple tool for predicting mortality in patients with ACS. It was described in 2004. This is derived from the largest multinational registry and includes the complete spectrum of patients with ACS, based on independent predictors of outcome. The 6-month GRACE risk score was developed and validated with more than 20000 patients, between 1999 and 2003, who were included in the GRACE registry (14 countries, 94 hospitals). This score allows us to calculate the probability of mortality during the first 6 months after discharge in all ACS spectrum (c-index higher than 0.70). The application of the GRACE risk score at admission was recommended by the clinical practice guidelines for risk stratification in ACS. The 6-month GRACE risk score was validated entirely across the wide range of current patients with $\mathrm{ACS}^{12}$ and demonstrated superiority over other risk scores. Its predictive value was also further validated over 6 months (even in the 5 -year follow-up). ${ }^{13}$

$\mathrm{CHA}_{2} \mathrm{DS}_{2} \mathrm{VASc}^{9}$ is a simple risk stratification schema to determine thromboembolic risk in patients with nonvalvular AF. It was validated in 2009; 5333 ambulant and hospitalised patients with AF were enrolled from 2003 to 2004. The $\mathrm{CHADS}_{2}$ is commonly used to assess risk of stroke, but $\mathrm{CHA}_{2} \mathrm{DS}_{2} \mathrm{VASc}$ scores have better discrimination of stroke risk, particularly in low-risk patients. Clinical practice guidelines recommended calculation of $\mathrm{CHA}_{2} \mathrm{DS}_{2} \mathrm{VASc}$ risk scores to determine whether patients with non-valvular AF need antithrombotic therapies for the prevention of stroke and systemic embolisation.

To date, no risk scores specifically estimate the risk of stroke after ACS. However, GRACE and $\mathrm{CHA}_{2} \mathrm{DS}_{2} \mathrm{VASc}$ risk scores could be useful in this setting. Our trial demonstrated that the accuracy of GRACE risk score was similar to the $\mathrm{CHA}_{2} \mathrm{DS}_{2} \mathrm{VASc}$ score to predict post-ACS 
stroke. This is important because with only one tool, which was initially designed to assess thrombotic risk, we can predict thromboembolic risk after ACS. We can identify patients who will benefit from a more potent antithrombotic treatment at discharge. Although our results are striking, they are in line with other studies previously published, such as Barra et $a .^{23}$ Also this observational retrospective single-centre cohort study, with fewer patients $(\mathrm{n}=1.711$ patients and post-ACS stroke rate of $4.3 \%$ ) and a shorter follow-up (median $17.4 \pm 8.7$ months), shows great predictive ability of the 6-month GRACE risk score (c-index $0.782 \pm 0.019$ ). In our cohort, we showed there was no difference between GRACE and $\mathrm{CHA}_{2} \mathrm{DS}_{2} \mathrm{VASc}$ risk scores to predict the risk of stroke after ACS. We have identified the GRACE risk score as a new independent predictor of stroke post-ACS.

The stroke has been considered a complication after $\mathrm{ACS}$; its frequency is especially increased in the first few months. ${ }^{19}$ One of the possible reasons that the GRACE risk score could predict the risk of stroke post-ACS is because many variables were previously demonstrated as independent predictors of post-ACS stroke. Thus, in recent years, predictors of post-ACS stroke have been identified in several trials: elderly, female sex, heart failure, coronary heart disease, AF, prior stroke, diabetes mellitus, timely revascularisation therapy, secondary prevention therapies and renal function. ${ }^{4} 618224-29$

Advanced age, a variable with great weightage in GRACE risk score, has proven to be a powerful risk factor for stroke in this population. ${ }^{2}{ }^{28}$ Several reports have shown that impaired renal function is also an efficient predictor of stroke and systemic embolism, and inhospital mortality of these patients. This was recently validated by Piccini et al in the ROCKET AF and ATRIAL Study Cohorts. ${ }^{22}$ Both elevated heart rate (more than $100 \mathrm{bpm})^{28}{ }^{30}$ as well as a high Killip class ${ }^{17}$ at admission have demonstrated increased mortality, reinfarction as well as the greater possibility of post-ACS stroke. Also, the ST-segment changes on index ECG, specially STEMI, predicts more events. ${ }^{31}$ Likewise, the value of GRACE at admission, GRACE risk score and higher markers of myocardial damage are predictors to stroke post-ACS. ${ }^{17} 31$ Percutaneous coronary intervention during hospitalisation predicts a decreased risk of ischaemic stroke; ${ }^{30}$ Van De Graaff et al ${ }^{6}$ showed a significant relationship between timely revascularisation therapy and risk of inhospital ischaemic stroke. The patients with previous coronary heart disease and especially anterior myocardial infarction showed an increase in post-ACS stroke. ${ }^{3}$ Heart failure and reduced left ventricular ejection fraction ${ }^{4} 1832$ had stronger association with mortality and also have been associated with increased risk of stroke after ACS. Because of this, although the GRACE score was not designed to determine the risk of stroke, it has good discriminative ability and a good correlation with $\mathrm{CHA}_{2} \mathrm{DS}_{2} \mathrm{VASc}$ to predict the primary end point. GRACE risk score included some variables which have not been included in the $\mathrm{CHA}_{2} \mathrm{DS}_{2} \mathrm{VASc}$ score. The high-risk GRACE score patients are usually older patients with renal failure and more Killip; therefore, these patients could be at higher risk of stroke.

\section{Clinical implications}

In recent years, there has been increasing interest to show the risk of stroke after ACS, and the risk factors associated with it. The GRACE risk score could eventually contribute to a better risk stratification and help us make decisions about interventions to reduce stroke after ACS in high-risk patients. Nowadays, the estimation of cardiovascular risk and individualisation has become a priority. GRACE risk score is used in routine clinical practice for risk stratification to optimise the treatment. Our study shows a new utility to predict stroke in the follow-up post-ACS.

\section{Limitations}

These data must be interpreted in the context of this study's limitations. It is a retrospective analysis of clinical single centre data. Our small-sized sample should be considered the main limitation of this study. In fact, the relatively low absolute number of ischaemic stroke events during follow-up reinforces the need for more studies with larger cohorts of patients to confirm the usefulness of GRACE risk score. However, irrespective of its potential future clinical validation, our study has supported the applicability of GRACE risk score in patients with ACS prior to discharge. Moreover, as many patients who died during follow-up were not autopsied or previously observed at the emergency department, it is very hard, if not impossible in some cases, to know whether stroke was the cause of death. Therefore, the true incidence of stroke was probably underestimated.

\section{Conclusions}

The GRACE model is a clinical risk score that facilitates the identification of individual patients who are at high risk of stroke after ACS discharge. New therapeutic interventions that have the potential to limit preventable post-ACS stroke may have the greatest impact on this vulnerable population.

Acknowledgements The authors would like to thank all the staff members (physicians, nurses and auxiliary members) and fellows from the cardiology department and coronary care unit for their support.

Competing interests None.

Provenance and peer review Not commissioned; externally peer reviewed.

Data sharing statement No additional data are available.

Open Access This is an Open Access article distributed in accordance with the Creative Commons Attribution Non Commercial (CC BY-NC 4.0) license, which permits others to distribute, remix, adapt, build upon this work noncommercially, and license their derivative works on different terms, provided the original work is properly cited and the use is non-commercial. See: http:// creativecommons.org/licenses/by-nc/4.0/ 


\section{REFERENCES}

1. Mooe $T$, Eriksson $P$, Stegmayr B. Ischemic stroke after acute myocardial infarction. Stroke 1997;28:762-7.

2. Witt BJ, Brown RD Jr, Jacobsen SJ, et al. A community-based study of stroke incidence after myocardial infarction. Ann Intern Med 2005;143:785-92.

3. Tanne D, Goldbourt U, Zion M, et al. Frequency and prognosis of stroke/TIA among 4808 survivors of acute myocardial infarction. The SPRINT Study Group. Stroke 1993;24:1490-5.

4. Witt BJ, Ballman KV, Brown RD Jr, et al. The incidence of stroke after myocardial infarction: a meta-analysis. Am J Med 2006;119:354.e1-9.

5. Budaj A, Flasinska K, Gore JM, et al. Magnitude of and risk factors for in-hospital and postdischarge stroke in patients with acute coronary syndromes: findings from a global registry of acute coronary events. Circulation 2005;111:3242-7.

6. Van De Graaff E, Dutta M, Das P, et al. Early coronary revascularization diminishes the risk of ischemic stroke with acute myocardial infarction. Stroke 2006;37:2546-51.

7. Maggioni AP, Franzosi MG, Santoro E, et al. The risk of stroke in patients with acute myocardial infarction after thrombolytic and antithrombotic treatment. N Engl J Med 1992;327:1-6.

8. Gage BF, Waterman AS, Shannon W, et al. Validation of clinical, classification schemes for predicting stroke: results from the National Registry of Atrial Fibrillation. JAMA 2001;285:2864-70.

9. Lip GY, Nieuwlaad R, Pisters R, et al. Refining clinical risk stratification for predicting stroke and thromboembolism in atrial fibrillation using a novel risk factor-bases approach: the euro heart survey on atrial fibrillation. Chest 2010;137:263-72.

10. Poçi D, Hartford M, Karisson T, et al. Role of the CHADS2 score in acute coronary syndromes. Risk of subsequent death or stroke in patients with and without atrial fibrillation. Chest 2012;141:1431-40.

11. Eagle KA, Lim MJ, Dabbous $\mathrm{OH}$, et al.; GRACE Investigators. $A$ validated prediction model for all forms of acute coronary syndrome: estimating the risk of 6-month postdischarge death in an international registry. JAMA 2004;291:2727-33.

12. Abu-Assi E, Ferreira-Gonzalez I, Ribera A, et al. Do GRACE (global registry of acute coronary events) risk scores still maintain their performance for predicting mortality in the era of contemporary management of acute coronary syndromes? Am Heart $J$ 2013;160:826-34, e821-3.

13. Fox KA, Carruthers KF, Dunbar DR, et al. Underestimated and under-recognized. The late consequences of acute coronary syndrome (GRACE UK-Belgium study). Eur Heart $J$ 2010;31:2755-64.

14. Fine JP, Gray R. A proporcional hazards model for the subdistrion of a competing risk. J Am Stat Assoc 1999;94:496-509.

15. Hanley JA, McNeil BJ. The meaning and use of the are under a receiver operating characteristic (ROC) curve. Radiology 1982;143:29-36.

16. Pencina MJ, D'Agostino RB Sr, D'Agostino RB Jr, et al. Evaluating the added predictive ability of a new marker: from area under the ROC curve to reclassification and beyond. Stat Med 2008;27:157-72.

17. Barra S, Providência R, Lourenço Gomes $P$, et al. Predicão do risco de evento cerebrovascular após um enfarte agudo de miocárdio. Rev Port Cardiol 2011;30:655-63.

18. Brammås A; Jakobsson S; Ulvenstam A, et al. Mortality after ischemic stroke in patients with acute myocardial infarction. Predictors and trends over time in Sweden. Stroke 2013;44:3050-5.
19. Mooe T, Olofsson BO, Eriksson P. Ischemic stroke. Impact of a recent myocardial infarction. Stroke 1999;30:997-1001.

20. Saczynski JS, Spencer FA, Gore JM, et al. Twenty-year trends in the incidence rates of stroke complicating acute myocardial infarction: Worcester Heart Attack Study. Arch Intern Med 2008;168:2104-10

21. Steg PG, James SK, Atar D, et al:; The Task Force on the management of ST-segment elevation acute myocardial infarction of the European Society of Cardiology (ESC). ESC Guidelines for the management of acute myocardial infarction in patients presenting with ST-segment elevation. Eur Heart $J$ 2012;33:2569-619.

22. Hamm CW, Bassand JP, Agewall S, et al.; ESC Committee for Practice Guidelines. ESC Guidelines for the management of acute coronary syndromes in patients presenting without persistent ST-segment elevation. The Task Force for the management of acute coronary syndromes (ACS) in patients presenting without persistent ST-segment elevation of the European Society of Cardiology (ESC). Eur Heart J 2011;32:2999-3054.

23. Barra S, Almeida I, Caetano F, et al. Stroke prediction with an adjusted R-CHA2DS2VASc score in a cohort of patients with a Myocardial Infartion. Thromb Res 2013;132:293-9.

24. Piccini JP, Stevens SR, Chang $Y$, et al. Renal dysfunction as a predictor of stroke and systemic embolism in patients with nonvalvular atrial fibrillation: validation of the R2CHADS2 Index in the ROCKET AF (Rivaroxaban Once-daily, oral, direct factor Xa inhibition Compared with vitamin $\mathrm{K}$ antagonism for prevention of stroke and Embolism Trial in Atrial Fibrillation) and ATRIA

(AnTicoagulation and Risk factors In Atrial fibrillation) study cohorts. Circulation 2013;127:224-32.

25. Providencia R, Fernandes A, Paiva L, et al. Decreased glomerular filtration rate and markers of left atrial stasis in patients with nonvalvular atrial fibrillation. Cardiology 2013;124:3-10.

26. Holzmann MJ, Aastveit A, Hammar N, et al. Renal dysfunction increases the risk of ischemic and hemorrhagic stroke in the general population. Ann Med 2012;44:607-15.

27. Mostofsky E, Wellenius GA, Noheria A, et al. Renal function predicts survival in patients with acute ischemic stroke. Cerebrovasc Dis 2009;28:88-94.

28. Wienbergen $\mathrm{U}$, Weber $\mathrm{MA}$, Muller $\mathrm{CH}$, et al. Incidence, risk factors, and clinical outcome of stroke after acute myocardial infarction in clinical practice: mir and mitra study groups: myocardial infarction registry: maximal individual therapy in acute myocardial infarction. Am J Cardiol 2001;87:782-5, A788.

29. [No authors listed]. Randomized trial of intravenous streptokinase, oral aspirin, both, or neither among 17,187 cases of suspected acute myocardial infarction: ISIS-2. ISIS-2 (Second International Study of Infarct Survival) Collaborative Group. J Am Coll Cardiol 1988;12(6 Suppl A):3A-13A.

30. Sampson UK, Pfeffer MA, McMurray JJ, et al. Predictors of stroke in high-risk patients after acute myocardial infarction: insights from the VALIANT trial. Eur Heart J 2007;28:685-91.

31. Park KL, Budaj A, Goldberg RJ, et al. Grace Investigators. Risk-prediction model for ischemic stroke in patients hospitalized with an acute coronary syndrome (from the global registry of acute coronary events [GRACE]. Am J Cardiol 2012;110:628-35.

32. Jakobsson S, Bergström L, Björklund F, et al. Risk of ischemic stroke after an acute myocardial infarction in patients with diabetes mellitus. Circ Cardiovasc Qual Outcomes 2014;7:95-101. 